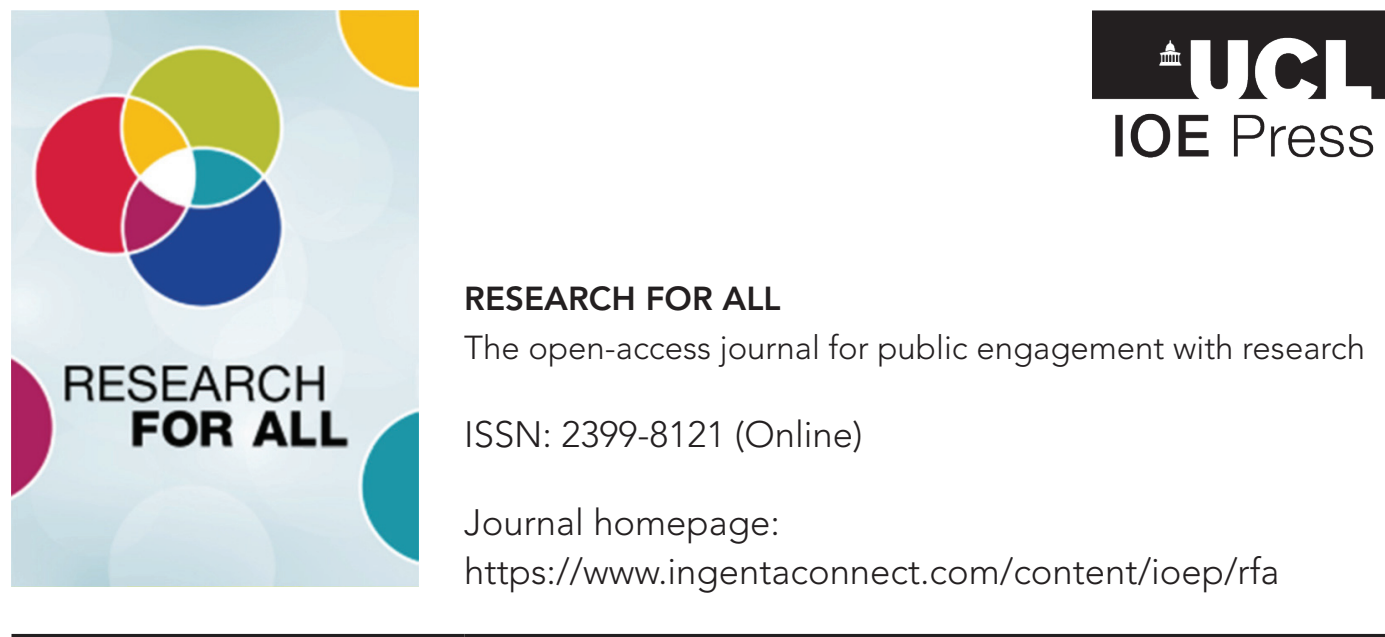

\title{
Reaching non-specialist audiences and engaging them with science at an affordable seaside campsite
}

Anna Woolman

\section{How to cite this article}

Woolman, A. (2020) 'Reaching non-specialist audiences and engaging them with science at an affordable seaside campsite'. Research for All, 4 (1): 6-15. DOI https://doi.org/10.18546/RFA.04.1.02

Submission date: 5 April 2019

Acceptance date: 9 October 2019

Publication date: 1 February 2020

\section{Peer review}

This article has been peer reviewed through the journal's standard double-blind peer review, where both the reviewers and authors are anonymized during review.

\section{Copyright}

CCopyright 2020 Woolman. This is an Open Access article distributed under the terms of the Creative Commons Attribution Licence (CCBY) 4.0 https://creativecommons.org/licenses/by/4.0/, which permits unrestricted use, distribution and reproduction in any medium, provided the original author and source are credited.

\section{Open access}

Research for All is a peer-reviewed open-access journal. 


\title{
Reaching non-specialist audiences and engaging them with science at an affordable seaside campsite
}

\author{
Anna Woolman* - British Science Association, UK
}

\begin{abstract}
In 2018, the British Science Association piloted a project that involved partnering with an activity provider and campsite in Ramsgate to determine whether affordable, accessible campsites near the seaside would be an effective location to reach audiences unengaged with science and engage them with food sustainability and eating insects. Results suggested that mostly unengaged audiences were present and that their attitude towards science improved after taking part. Future work should trial different activities and campsite partnerships across the UK, on a larger scale and at busier times of year.
\end{abstract}

Keywords: campsite, public engagement, insects, informal learning, food, entomology

\section{Key messages}

- Affordable campsites near the seaside are likely to be an effective way to reach audiences who do not make an active effort to engage with science.

- Campsites are ideal locations for extended engagement (that is, dwell time of $10+$ minutes).

- Campsites are particularly busy in the run-up to the bank holidays and summer months (school holidays), and need a long period of notice to support organizers to run activities during this time, but doing so will likely mean a larger audience for activities.

\section{Introduction}

The British Science Association (BSA) aims to make science a fundamental part of society and culture (BSA, n.d. a). As part of this, a key priority is developing programmes and activities that are most likely to engage non-specialist audiences. These are referred to as 'inactive' and 'not interested' in scientific research on the BSA's audience model (Figure 1). This is used internally to signpost such audiences to further activity, enabling them to identify as 'engaged' and/or 'professionals' should they choose, without expecting them, or forcing them, to move along the model. As a result of years of experience in the public engagement sector, and with an awareness of the fact that the general public are not one homogeneous group, since 2015 the BSA has been developing the model to better understand how engaged UK audiences are with 
science (Mendick and Sheldrake, 2016). Research from 2016 by King's College London suggested that 'not interested' and 'inactive' audiences account for 76 per cent of the UK adult population (BSA, n.d. b). Typically, these audiences are difficult to engage with traditional science communication activities (such as lectures and debates), largely because these formats rely on audiences making an active decision to physically go to them. Therefore, other avenues are usually explored, such as science busking in town centres and activities in shopping malls and community festivals, which focus more on bringing the event or activity directly to where such audiences may be (Wellcome Trust, 2014). However, independent holiday locations such as camp or caravan sites have been largely overlooked as a potential engagement space.

Figure 1: The BSA's audience model is used to determine an event participant's engagement with science - audiences classed as 'not interested' or 'inactive' have a lower engagement with science, whereas 'engaged' and 'professionals' audiences have a higher engagement with science (BSA, n.d. b)

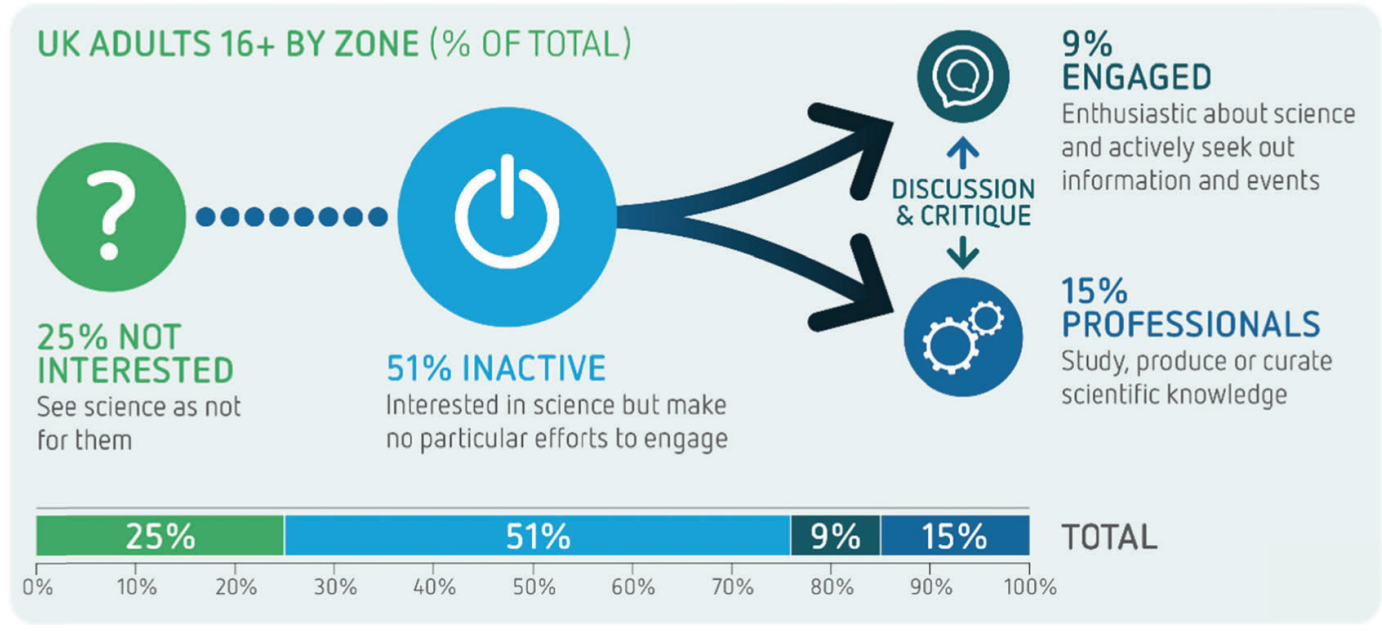

Source: King's College London 'Culture Tracker' 2016, which questioned a representative sample of UK adults about their relationship with science.

In general, audiences from lower socio-economic backgrounds have lower rates of engagement with activities traditionally described as 'cultural', such as visiting museums and galleries (Warwick Commission, 2015). It is not that these audiences do not necessarily want to engage, but that they experience a range of barriers to participation. For example, certain groups may feel as though these spaces are not for them (Dawson, 2018). As a result, other activities, such as the family holiday, or a trip to a cinema, which are familiar and can guarantee a 'good time', take priority (Audience Agency, 2019). Taking consideration of these factors, in recent years some science engagement programmes have shifted towards bringing the activities to where the audiences are, as opposed to expecting them to come to the activities (Woolman, 2018; STEM Hub, 2018), and this project aimed to be part of this shift.

The Audience Agency (2019) suggests that audiences from low socio-economic backgrounds who have low engagement with 'high' cultural activities, which can include those relating to science (Warwick Commission, 2015), are more likely to have affordable, low-cost holidays that involve trips to the UK seaside or holiday camps/ resorts. Yet, aside from the partnership between Butlin's (a large UK chain of seaside resorts) and the Royal Institution for their Science Zones that targeted children (Royal Institution, 2017) and their Family Science Weekend (Pope, 2016), there is no evidence 
that campsites or holiday parks regularly provide science activities, let alone for adults. Using accommodation cost as a proxy for a holidaymaker's engagement with science, this pilot project aimed to trial the effectiveness of an affordable, independent campsite by the seaside as a way to reach and engage audiences, of all ages, who may have a 'not interested' or 'inactive' relationship with science (Figure 1). The project's activities centred around the topic of insects as a sustainable food source, involving an entomologist and local activity provider to deliver them. Although participant number was relatively small (26 in total), results offer a promising insight into such campsites being a means to reach these audiences.

\section{Location, partnerships and timings}

In light of The Audience Agency's (2019) research referenced in the Introduction, the decision was made to focus on finding a campsite near to the seaside. To refine the 'search area', a partnership was formed with an activity provider in Ramsgate (a seaside town in Kent) with which the BSA had previous connections - Discovery Planet. This partner was chosen not only because of their proximity to the seaside and knowledge of the area, but also because of their track record of developing and delivering science activities for underserved audiences at a grassroots level. It was useful to work alongside them and have their support throughout the process.

Due to their knowledge of the local area, Discovery Planet were instrumental in researching and securing an appropriate campsite in the area that fitted the criteria (low cost, by the seaside and easily accessible by car/train).

The activity took place on 23 June 2018 and, for reasons of availability of all parties, it was timed to run between 10.00 a.m. and 4.00 p.m. The activities took place outside under a gazebo, in the middle of the campsite to ensure that all holidaymakers could see them.

\section{Activity}

Discovery Planet had previously developed and delivered an activity, Creepy Crawly Cuisine, which integrated insect ingredients into a number of foods, from soy baked mealworms, to banana bread with grubs in it, and cupcakes made from cricket flour. After discussions, it was decided that this activity would work well in the campsite setting. Some of the foods had very evident insect ingredients, but in other foods, the ingredients were difficult to distinguish, to make the food and activity more approachable to those who may be less confident to engage with the topic. The foods were presented in a similar way to how a traditional summer fete baking stall might look, on cake stands and with bunting (Figure 2 and Figure 3). It was important to make the stall inviting, especially considering that holidaymakers could choose to ignore the activity altogether, opting to go to the beach instead. People could stop by the stall in their own time. To ensure the target audience was reached, the activity was only available to those staying in the campsite and was free to take part; hence, no marketing materials were produced for distribution outside the campsite in the run-up.

Alongside the baked goods, Discovery Planet produced an insect fact sheet and hypothetical menu (Figure 4), which participants could take away with them. An entomologist, who had experience of researching insects as a potential food source, was also present to allow anyone who wanted to engage with the topic further a chance to discuss the issues raised by the activity. 
Figure 2: Photographs from the event showing how the foods made with insect ingredients were presented
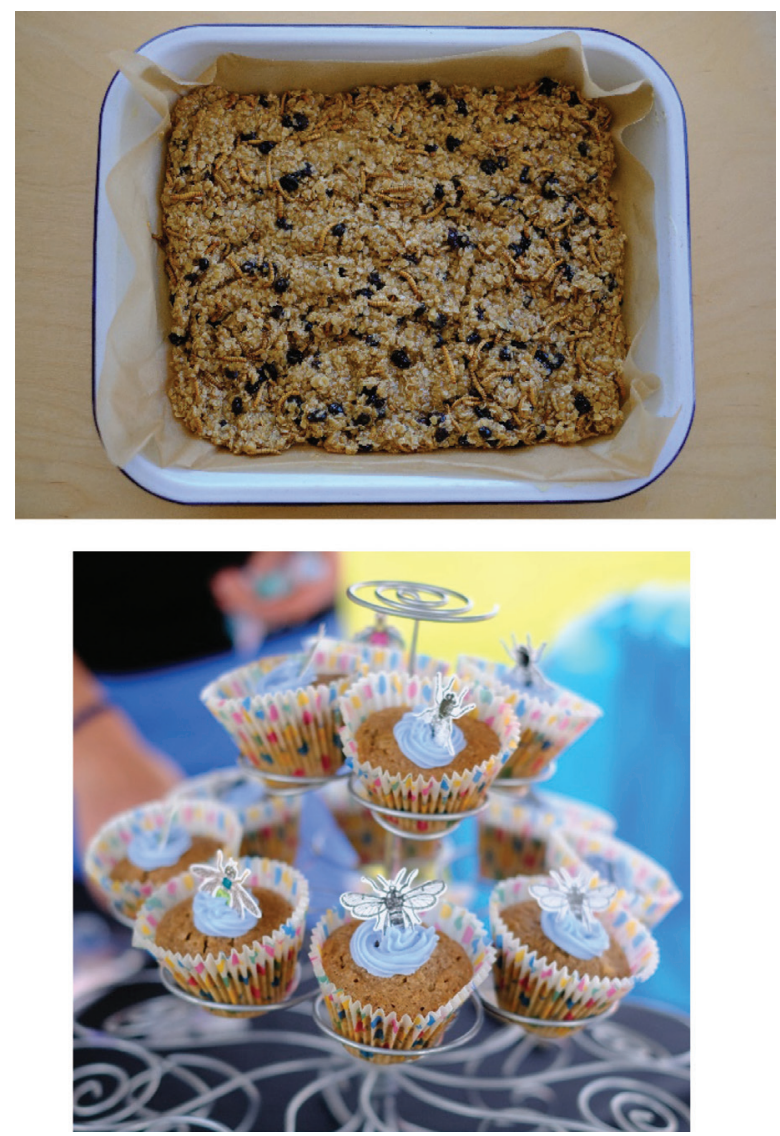

Credit: Pete Bateson, 2018

Figure 3: Participants at the campsite science activity

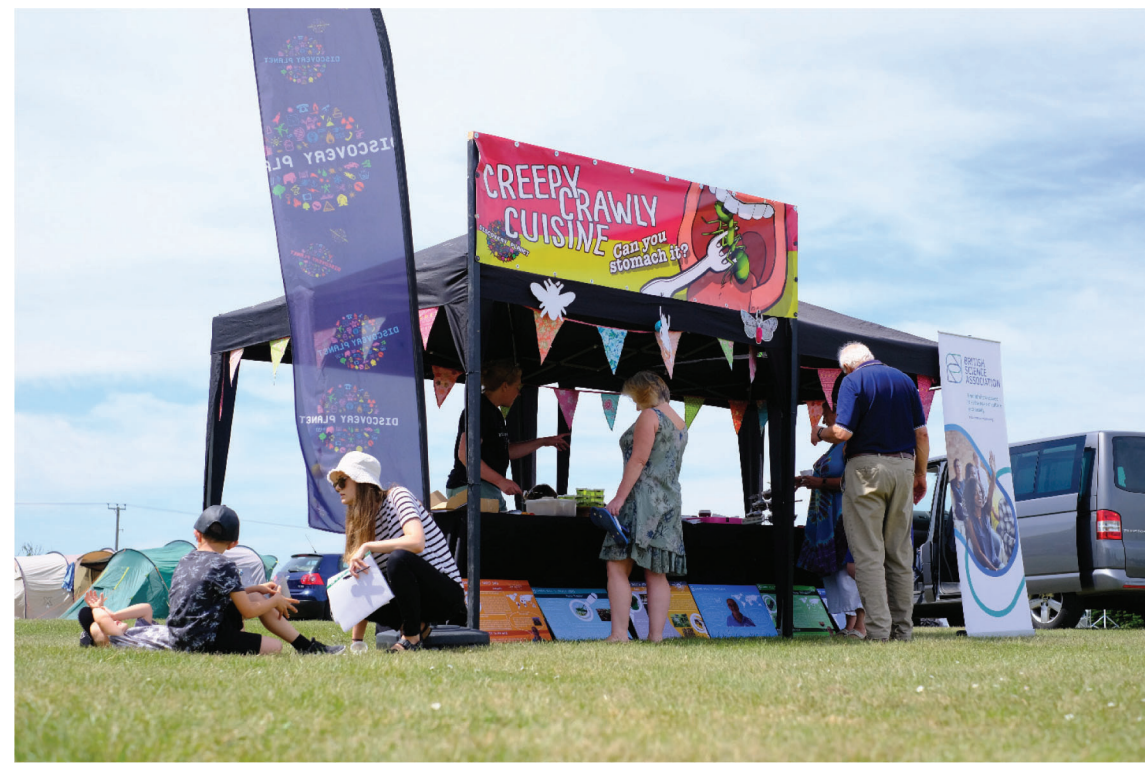

Credit: Pete Bateson, 2018 
Figure 4: The 'Creepy Crawly Cuisine' menu that Discovery Planet produced for the campsite activity

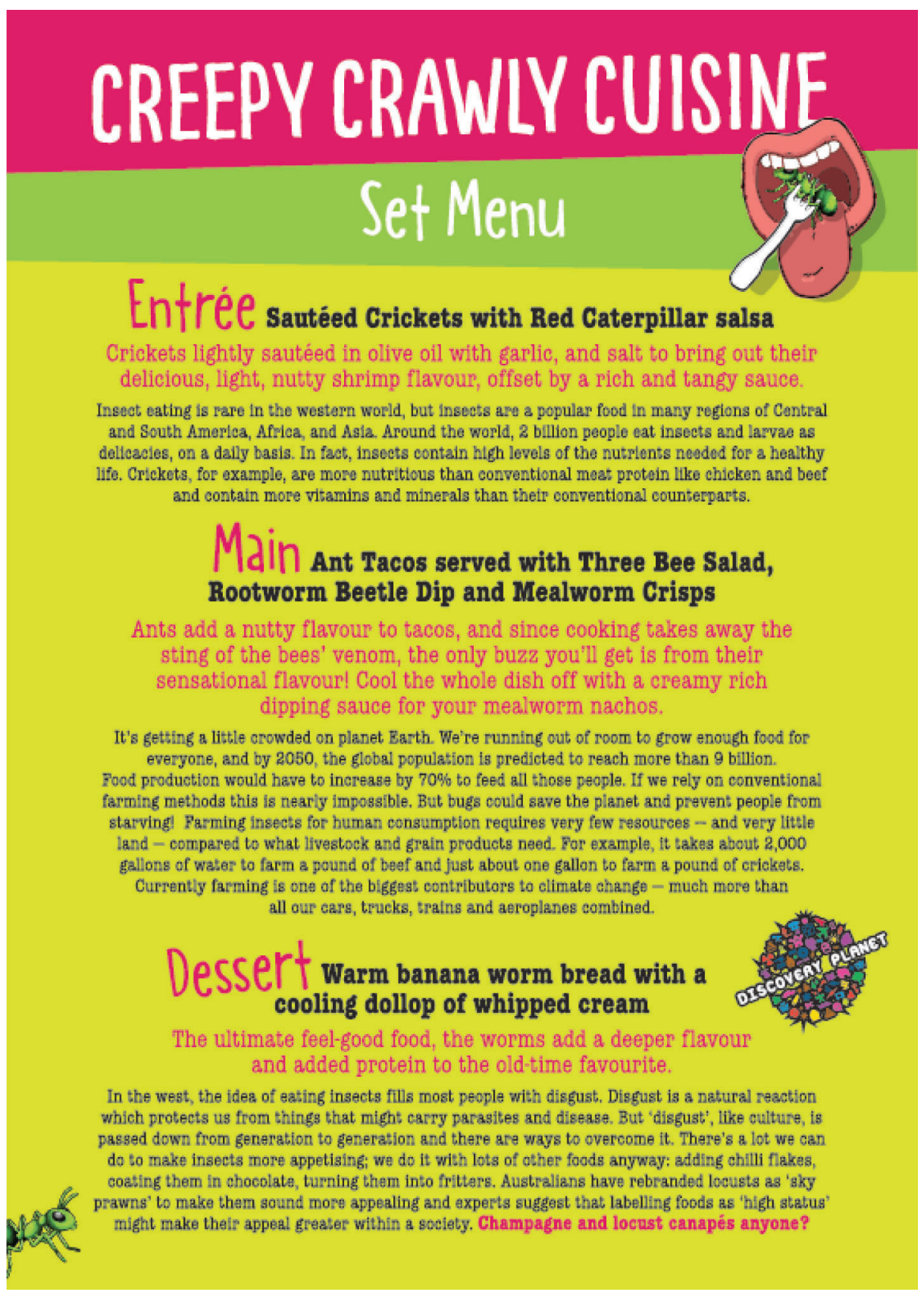

From observations, it was evident that the topic, insects as a sustainable food source, was something that provided intergenerational engagement. Adults, teenagers and children were all interested in the activity and gained something from it. Foods made of insect products are engaging because of the initial 'eww' factor. However, once people started to talk to the activity provider and entomologist, they had thoughtprovoking conversations about the future of food and attitudes towards eating insects. A topic involving insects worked well in this environment because of the fact that the activities were taking place outside, in a campsite. It would be interesting to see what other topics could work in a similar way in the future. 


\section{Key findings}

To obtain participant feedback and demographic data, paper self-reporting evaluation forms were given to the participants to complete. Anecdotal feedback from conversations with the campsite's management, Discovery Planet and the entomologist was used to gauge their experience of the event and receptivity to them taking part in such projects in the future.

To determine the audience's engagement with science before taking part in the activity, the following was asked in the evaluation form that they completed after taking part:

1. Do you work in a science related job? [YES = 'professionals'] If no, before this event which of these statements best described your relationship with science?

a) I feel connected with science - I actively seek out science news, events, activities or entertainment [YES = 'engaged']

b) I'm interested in science, but I don't make a special effort to keep informed [YES = 'inactive']

c) Science is not for me [YES = 'not interested']

\section{Audience experience}

There were 26 participants, and 11 of them completed an evaluation form. The small number of participants possibly benefited the engagement - all of the interactions lasted for 10+ minutes. While this may be partly due to the small number of attendees providing a relaxed environment for conversation, it is also likely that the location of a campsite fostered this amount of dwell time. As people were on holiday, they were relaxed and happy to spend extended periods of time engaged in an activity. This is in comparison to other common drop-in locations, such as a shopping centre or a high street, where people have other things to do and are less likely to spend long periods of time taking part in an activity that they were not expecting to be there.

All respondents rated the event as either 'excellent' or 'good'. The most commonly used word to describe the event was 'interesting', followed by 'different'. Positive comments were received from respondents of differing ages, which can be found below. Together, the feedback demonstrates the ability of this specific activity to effectively engage a wide range of age groups:

- 'Very friendly \& helpful' (age 35-9)

- 'I really enjoyed the event because it's so different to what I'm used to' (age 11-15)

- 'Tasty food. Really thought provoking' (age 50-4)

- 'Very interactive, great event' (age 11-15).

The majority of respondents were in the 'inactive' audience category, and no respondents were classified as 'professionals'. Respondents said that they were either a lot or a bit more interested in science as a result of the activity, with one person saying that their interest level was unchanged. This change in the majority of respondents' interest in science is mirrored in their responses to the question 'After attending this event, are you more likely to look out for and attend science events and activities in the future?', where all responded with 'definitely' or 'possibly'. However, whether these intentions translated into actions was not measured. Most attendees had also either not been to a science festival before or had been to only one. 


\section{Facilitator experience}

Discovery Planet: 'We had a brilliant day on Saturday, thoroughly enjoyable and I think we had some good quality chats to the visiting campers.' Anecdotal feedback from conversations with Discovery Planet suggested that they would consider campsites as a viable option to host activities in the future.

Campsite management: Conversations with the campsite manager in the run-up to the event, as well as on the day, demonstrated that they were excited to be hosting science activities and were glad about how everything went. They said they were open to hosting more activities of this nature.

Entomologist/researcher: Conversations with the researcher demonstrated that they had some interesting interactions with participants. While some participants shied away from the insect-eating activity and opted to engage in conversation about sustainability from the beginning, others were more reluctant to talk but after participating in the activity started asking questions about why and how insects could form part of a sustainable diet. The researcher was keen to take part in future activities of this nature, and mentioned that it provided insight to talk about the topic with people who may not normally engage with it, or science in general, in their day-to-day lives.

\section{Critical reflections}

\section{Number of participants and respondents}

Of the 26 participants, just under half completed an evaluation form $(n=11)$. The nature of the event, with it being drop-in and very informal, meant that it was hard to get participants to complete forms. In hindsight, it might have been better to conduct on-the-spot interviews, reduce the number of questions on the form or make certain questions (such as about people's engagement with science) more interactive.

It is likely that the small number of attendees was due to the activity taking place on 23 June, outside the campsite's peak holiday booking time. It also occurred during the day, from 10.00 a.m. to 4.00 p.m., due to the availability of various parties. However, it was suggested by campsite management that attendee numbers may have been larger if the event had taken place in the evening or earlier in the morning, when holidaymakers were more likely to be on site. It was warm and sunny for the duration of the event, so the weather was not a key factor, if at all, in the numbers of people interacting with the activity.

Holding the event outside peak holiday time was initially a strategic decision to gauge interest from the campsite and to trial the activities on a smaller audience. If it had taken place later in the holiday season, there would no doubt have been a larger audience. However, if this was to be repeated at a busier time of year, then discussions with the campsite would have to be started well in advance, ahead of when accommodation bookings are being taken for the holiday season. This would reduce pressure on management and ensure space is available for the activity in the campsite.

\section{Audience engagement}

The activity worked well, facilitating a long dwell time of 10+ minutes for the attendees. This long dwell time could also be attributed to the fact that those on holiday have a lot of leisure time to spend being involved with such activities (rather than in locations where science engagement activities typically take place, such as shopping centres, 
high streets and even at festivals). From this project, it is clear that holiday locations, such as affordable campsites, are places with lots of potential for informal, but meaningful, science engagement with the public.

This project was successful with a majority 'inactive' audience participating. Typically, this is uncommon for 'traditional' science engagement events, such as the British Science Festival (BSA, 2017), which is also organized by the BSA. Most of the UK population fall into the 'inactive' category, with less than 25 per cent of audiences in 'professionals' and 'engaged' categories (Figure 1), and so special considerations need to be made in how to effectively engage the former. Although the results from this pilot project are promising in terms of affordable campsites reaching and engaging 'inactive' audiences, further work needs to be done to obtain a more holistic understanding of the different audiences that campsites in different areas, and at different times of year, attract.

\section{Searching for the 'right' campsite}

Forming relationships, as well as finding relevant campsites that fit the criteria of being affordable and by the seaside, was harder than anticipated. This is because there is a wide variety of campsites and caravan parks across the UK, from affordable holiday parks to high-end campsites. Unsurprisingly, minimal data exist on the socio-economic status of people attending specific campsites in the UK, and it was also difficult to reach out to campsites without any prior experience of them, especially because the BSA is situated in central London. To begin with, large, affordable campsite chains were targeted but this proved unfruitful, mostly because such campsites already had an extensive programme of events.

Therefore, through the partnership with Discovery Planet, independent campsites in Ramsgate were identified and targeted instead. This not only refined the 'search area', but such campsites were also unlikely to have other events happening, and management were more responsive.

\section{Summary}

Overall, this pilot project successfully reached and engaged an audience who would otherwise not actively engage with science, increasing their interest levels.

The results of this project can at least be taken to suggest that affordable campsites near the seaside enable a broad range of people to be reached, with the majority likely to be from an 'inactive' science engagement background as per the BSA's audience model. Future iterations of this project could compare different holiday locations across the UK, and different times of year, times of day and scientific topics, to determine the most effective means to reach and engage 'inactive' and 'not interested' audiences in this way.

\section{Acknowledgements}

I would like to extend enormous gratitude to Xanthe Pitt and Nikki Hildesley from Discovery Planet who went above and beyond to provide invaluable input to the project. Without funding from the British Science Association this project would not have been possible, and I would like to thank Rosie Waldron, Ivvet Modinou and colleagues at the Association for their continued support throughout the process. Finally, thank you to the campsite owners and entomologist for their positive attitude and willingness to be involved. 


\section{Notes on the contributor}

Anna Woolman is Engagement Manager at the British Science Association. Having previously been Coordinator of the UKScience Festivals Network, she currently manages the British Science Festival public programme. Besides this, she has experience of working on a variety of engagement projects, from writing 'virtual race' scripts, to managing projects that reach people from backgrounds traditionally underserved by science engagement activities. She is particularly interested in exploring new ways to reach audiences who do not see science as 'for them'.

\section{References}

Audience Agency (2019) 'Facebook Families'. Online. https://tinyurl.com/yy33xlwp (accessed 2 November 2019).

BSA (British Science Association) (n.d. a) 'About us'. Online. https://tinyurl.com/ybtdyabf (accessed 8 April 2019).

BSA (British Science Association) (n.d. b) 'Our audience model'. Online. www.britishscienceassociation.org/our-audience-model (accessed 8 April 2019).

BSA (British Science Association) (2017) British Science Festival Evaluation Report. London: British Science Association. Online. https://tinyurl.com/yxjenydf (accessed 2 November 2019).

Dawson, E. (2018) 'Reimagining publics and (non) participation: Exploring exclusion from science communication through the experiences of low-income, minority ethnic groups'. Public Understanding of Science, 27 (7), 772-86.

Mendick, H. and Sheldrake, R. (2016) Transforming Science Engagement: The impact of the British Science Association's work. London: British Science Association. Online. https://tinyurl.com/y3yjq2mc (accessed 2 November 2019).

Pope, K. (2016) 'Butlins Astonishing Family Science Weekend: Review'. The Guardian, 9 March. Online. https://tinyurl.com/y2hhfkgu (accessed 2 November 2019).

Royal Institution (2017) 'Royal Institution and L'Oréal UK and Ireland to headline at Butlin's'. Royal Institution News, 3 March. Online. www.rigb.org/about/news/spring-2017/butlins (accessed 9 April 2019).

STEM Hub (2018) 'Margate Discovery Planet - Turbines: A wind-wind solution'. STEM Hub News, 4 September. Online. www.thestemhub.org.uk/index.php/news/news-article/183 (accessed 8 May 2019).

Warwick Commission (2015) Enriching Britain: Culture, creativity and growth: The 2015 report by the Warwick Commission on the Future of Cultural Value. Coventry: University of Warwick. Online. https://tinyurl.com/y58kjkm3 (accessed 2 November 2019).

Wellcome Trust (2014) Experiments in Engagement: Engaging with young people from disadvantaged backgrounds. London: Wellcome Trust. Online. https://tinyurl.com/y4odkns5 (accessed 2 November 2019).

Woolman, A. (2018) 'Engaging disadvantaged young people with science through the Network'. UK Science Festivals Network, 29 January. Online. https://tinyurl.com/yxqgn5pm (accessed 2 November 2019). 